\title{
Inference on q-Weibull parameters
}

DOI:

10.1007/s00362-017-0951-3

\section{Document Version}

Accepted author manuscript

Link to publication record in Manchester Research Explorer

\section{Citation for published version (APA):}

Jia, X., Nadarajah, S., \& Guo, B. (2017). Inference on q-Weibull parameters. Statistical Papers, 1-19. https://doi.org/10.1007/s00362-017-0951-3

\section{Published in:}

Statistical Papers

\section{Citing this paper}

Please note that where the full-text provided on Manchester Research Explorer is the Author Accepted Manuscript or Proof version this may differ from the final Published version. If citing, it is advised that you check and use the publisher's definitive version.

\section{General rights}

Copyright and moral rights for the publications made accessible in the Research Explorer are retained by the authors and/or other copyright owners and it is a condition of accessing publications that users recognise and abide by the legal requirements associated with these rights.

\section{Takedown policy}

If you believe that this document breaches copyright please refer to the University of Manchester's Takedown Procedures [http://man.ac.uk/04Y6Bo] or contact uml.scholarlycommunications@manchester.ac.uk providing relevant details, so we can investigate your claim.

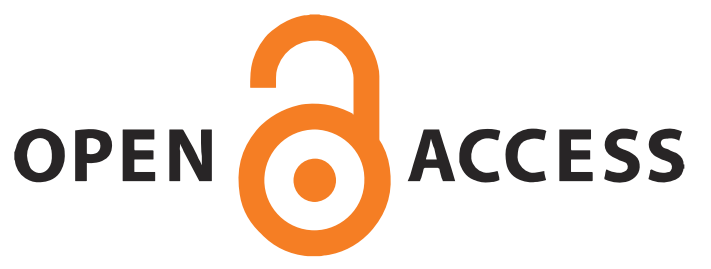




\title{
Inference on q-Weibull parameters
}

\author{
Xiang Jia ${ }^{1}$, Saralees Nadarajah ${ }^{2}$, Bo Guo ${ }^{1}$
}

1. College of Information System and Management, National University of Defence Technology, Hunan 410073, P.R. China

2. School of Mathematics, University of Manchester, Manchester M13 9PL, UK

\begin{abstract}
The q-Weibull distribution is a generalization of the Weibull distribution and could describe complex systems. We firstly point out how to derive the maximum likelihood estimates (MLEs) and least-squares estimates (LSEs) of the q-Weibull parameters. Next, three confidence intervals (CIs) for the q-Weibull parameters are constructed based on bootstrap methods and asymptotic normality of the MLEs. Explicit expressions for the Fisher information matrix necessary for the asymptotic CIs are derived. A Monte Carlo simulation study is conducted to compare the performances of the MLEs and LSEs as well as the different CIs. The simulation results show that the MLEs are superior to the LSEs in terms of both bias and mean squared error. The bootstrap CIs based on the MLEs are shown to have good coverage probabilities and average interval widths. Finally, a real data example is provided to illustrate the proposed methods.
\end{abstract}

Keywords: Confidence interval; Least-squares estimate; Maximum likelihood estimate

\section{Introduction}

Recently, q-type distributions have been introduced to describe complex systems in the context of nonextensive statistical mechanics. They have been widely applied in many fields, such as physics, chemistry, biology, mathematics, geography, economics, medicine, informatics and linguistics [1-3]. The q-type distributions are based on functions introduced in nonextensive statistical mechanics for nonextensive formalism. One of the functions is the q-exponential function defined as

$$
\exp _{q}(x)=\left\{\begin{array}{ll}
{[1+(1-q) x]^{\frac{1}{1-q}},} & 1+(1-q) x>0 \\
0, & \text { otherwise }
\end{array} .\right.
$$

Several q-type distributions have been proposed, including the q-exponential [3-6], q-gamma [5], q-Gaussian [3], q-Weibull [37] and others [5]. As the Weibull model is most commonly used to describe lifetime data [8,9], the corresponding q-type distribution, i.e. the q-Weibull distribution, has been more focused than others.

The q-Weibull distribution can describe complex systems with long-range interactions and long-term memory [10]. The Weibull distribution can only exhibit monotonic and constant shapes for its hazard rate function. However, the q-Weibull distribution can exhibit unimodal, bathtub-shaped, monotonically decreasing, monotonically increasing and constant shapes [7, 10] for its hazard rate function. Hence, it is a useful generalization of the Weibull distribution [11]. The q-Weibull distribution was considered with the autoregressive conditional duration model [12, 13], stress-strength model [14] and maxmin processes [15]. Besides, it has been used to analyse data for dielectric breakdown regime of ultra-thin oxides [16], natural gas recovery plants [17] and robotic welding stations [18].

In applications, the most basic problem is accurate estimation of the q-Weibull parameters. Several methods have been proposed to estimate the q-Weibull parameters, including moment estimates [14], maximum likelihood estimates (MLEs) [10] and least-squares estimates (LSEs) [7, 16, 17]. The confidence intervals (CIs) for the q-Weibull parameters were considered only by Xu et al. [10]. These developments motivate us to compare the different estimates and propose CIs for the q-Weibull parameters.

This study has two objectives. First, it provides clear derivations of the MLEs and LSEs of the q-Weibull parameters and compares their performance through a simulation study. The second objective is to construct CIs for the q-Weibull parameters and compare their performance.

The rest of the paper is organised as follows. The q-Weibull distribution and its MLEs and LSEs are introduced in Section 2. Different CIs are constructed in Section 3. In Section 4, a simulation study is conducted to compare the MLEs and LSEs and also to compare the different CIs. A real data example is presented in Section 5. The paper is concluded in Section 6. 


\section{Estimation of the q-Weibull parameters}

Throughout, we assume complete data sets are available from the q-Weibull distribution. That is, no censoring is considered. Furthermore, the minimum life parameter is considered null throughout.

\subsection{The q-Weibull distribution}

The probability density function (PDF) of the q-Weibull distribution is

$$
f_{q}(t)=(2-q) \frac{\beta}{\eta}\left(\frac{t}{\eta}\right)^{\beta-1} \exp _{q}\left[-\left(\frac{t}{\eta}\right)^{\beta}\right],
$$

where $\beta>0$ and $q<2$ are shape parameters, and $\eta>0$ is a scale parameter.

By introducing $\lambda=\eta^{-\beta}$ and using $\exp _{q}(x)$ in Eq. (1), the PDF of the q-Weibull distribution can be rewritten as

$$
f_{q}(t)=(2-q) \lambda \beta t^{\beta-1}\left[1-(1-q) \lambda t^{\beta}\right]^{\frac{1}{1-q}},
$$

where

$$
t \in\left\{\begin{array}{ll}
{[0,+\infty),} & 1<q<2, \\
{\left[0,[\lambda(1-q)]^{-\frac{1}{\beta}}\right],} & q<1
\end{array} .\right.
$$

The limiting PDF for $q=1$ is

$$
\begin{aligned}
\lim _{q \rightarrow 1} f_{q}(t) & =\lambda \beta t^{\beta-1} \lim _{q \rightarrow 1}\left\{\left[1-(1-q) \lambda t^{\beta}\right]^{\frac{-1}{(1-q) \lambda t^{\beta}}}\right\}^{-\lambda t^{\beta}} \\
& =\lambda \beta t^{\beta-1} \exp \left(-\lambda t^{\beta}\right)
\end{aligned}
$$

a Weibull PDF. The cumulative distribution function (CDF) of the q-Weibull distribution is

$$
F_{q}(t)=1-\left[1-(1-q) \lambda t^{\beta}\right]^{\frac{2-q}{1-q}}
$$

If $q<1$,

$$
\begin{aligned}
E\left(T^{s}\right) & =\int_{0}^{[\lambda(1-q)]^{-\frac{1}{\beta}}} t^{s}(2-q) \lambda \beta t^{\beta-1}\left[1-(1-q) \lambda t^{\beta}\right]^{\frac{1}{1-q}} d t \\
& =\frac{2-q}{(1-q)^{1+\frac{s}{\beta}} \lambda^{\frac{s}{\beta}}} B\left(\frac{s}{\beta}+1, \frac{2-q}{1-q}\right),
\end{aligned}
$$

where

$$
B(x, y)=\int_{0}^{1} t^{x-1}(1-t)^{y-1} d t=\int_{0}^{+\infty} \frac{t^{x-1}}{(1+t)^{x+y}} d t
$$

denotes the beta function. It follows that the mean and variance of the q-Weibull random variable when $q<1$ are

$$
\begin{aligned}
E(T) & =\frac{2-q}{(1-q)^{1+\frac{1}{\beta}} \lambda^{\frac{1}{\beta}}} B\left(\frac{1}{\beta}+1, \frac{2-q}{1-q}\right), \\
\operatorname{Var}(T) & =\frac{2-q}{(1-q)^{1+\frac{2}{\beta}} \lambda^{\frac{2}{\beta}}}\left[B\left(\frac{2}{\beta}+1, \frac{2-q}{1-q}\right)-\frac{2-q}{1-q} B^{2}\left(\frac{1}{\beta}+1, \frac{2-q}{1-q}\right)\right] .
\end{aligned}
$$

If $1<q<2$,

$$
\begin{aligned}
E\left(T^{s}\right) & =\int_{0}^{+\infty} t^{s}(2-q) \lambda \beta t^{\beta-1}\left[1+(q-1) \lambda t^{\beta}\right]^{-\frac{1}{q-1}} d t \\
& =\frac{2-q}{(q-1)^{1+\frac{s}{\beta}} \lambda^{\frac{s}{\beta}}} B\left(\frac{s}{\beta}+1, \frac{1}{q-1}-\frac{s}{\beta}-1\right)
\end{aligned}
$$


provided $\frac{1}{q-1}-\frac{s}{\beta}>1$. It follows that the mean and variance of the $\mathrm{q}$-Weibull random variable when $1<q<2$ are

$$
\begin{aligned}
E(T) & =\frac{2-q}{(q-1)^{1+\frac{1}{\beta}} \lambda^{\frac{1}{\beta}}} B\left(\frac{1}{\beta}+1, \frac{1}{q-1}-\frac{1}{\beta}-1\right) \\
\operatorname{Var}(T) & =\frac{2-q}{(q-1)^{1+\frac{2}{\beta}} \lambda^{\frac{2}{\beta}}}\left[B\left(\frac{2}{\beta}+1, \frac{1}{q-1}-\frac{2}{\beta}-1\right)-\frac{2-q}{q-1} B^{2}\left(\frac{1}{\beta}+1, \frac{1}{q-1}-\frac{1}{\beta}-1\right)\right]
\end{aligned}
$$

provided $\frac{1}{q-1}-\frac{1}{\beta}>1$ and $\frac{1}{q-1}-\frac{2}{\beta}>1$.

\subsection{The MLEs of the q-Weibull parameters}

is

Let $t_{1}, \ldots, t_{n}$ be a random sample from the q-Weibull distribution. The logarithm of the likelihood function for this sample

$$
\ln L=n \ln (2-q)+n \ln \beta+n \ln \lambda+(\beta-1) \sum_{i=1}^{n} \ln t_{i}+\frac{1}{1-q} \sum_{i=1}^{n} \ln \left[1-(1-q) \lambda t_{i}^{\beta}\right] .
$$

The first derivatives of the log-likelihood function with respect to $q, \beta$ and $\lambda$ are

$$
\begin{aligned}
\frac{\partial \ln L}{\partial q} & =\frac{n}{q-2}+\frac{\lambda}{1-q} \sum_{i=1}^{n} \frac{t_{i}^{\beta}}{1-(1-q) \lambda t_{i}^{\beta}}+\frac{1}{(1-q)^{2}} \sum_{i=1}^{n} \ln \left[1-(1-q) \lambda t_{i}^{\beta}\right], \\
\frac{\partial \ln L}{\partial \beta} & =\frac{n}{\beta}+\sum_{i=1}^{n} \ln t_{i}-\sum_{i=1}^{n} \frac{\lambda t_{i}^{\beta} \ln t_{i}}{1-(1-q) \lambda t_{i}^{\beta}}, \\
\frac{\partial \ln L}{\partial \lambda} & =\frac{n}{\lambda}-\sum_{i=1}^{n} \frac{t_{i}^{\beta}}{1-(1-q) \lambda t_{i}^{\beta}} .
\end{aligned}
$$

The derivatives are nonlinear. So, closed-form solutions for the MLEs are difficult to obtain. Hence, the MLEs $\widehat{q}_{m}, \widehat{\beta}_{m}$ and $\widehat{\lambda}_{m}$ are computed by the following constrained optimization problem

$$
\begin{array}{cc}
\max & \ln L \\
\text { s.t. } & q<2, \\
& \lambda>0, \\
& \beta>0,
\end{array}
$$

where $\ln L$ is given by (5). It is difficult to prove the uniqueness of the solution to the optimization problem (6). An optimization algorithm described later on was applied to solve this optimization problem. Execution of the algorithm for a wide range of initial values of the parameters showed that the algorithm always converged and the solution was unique. The initial values considered corresponded to all combinations of $q=0,0.2, \ldots, 1.8, \lambda=1,2, \ldots, 10$ and $\beta=1,2, \ldots, 10$.

For the objective function $\ln L, \ln \left[1-(1-q) \lambda t_{i}^{\beta}\right]$ exists for $i=1, \ldots, n$. The constraints $1-(1-q) \lambda t_{i}^{\beta}>0$ should be accounted for the optimization problem (6). However, $t_{i}>0$ when $1<q<2$ and $0<t_{i}<[\lambda(1-q)]^{-\frac{1}{\beta}}$ when $q<1$. Hence, $1-(1-q) \lambda t_{i}^{\beta}>0$ always holds and the constraints can be ignored.

\subsection{The LSEs of the q-Weibull parameters}

The $\mathrm{CDF} F_{q}(t)$ in (4) can be rewritten as

$$
\begin{aligned}
F_{q}(t) & =1-\left[1-\left(1-\frac{1}{2-q}\right) \lambda(2-q) t^{\beta}\right]^{\frac{1}{1-\frac{1}{2-q}}} \\
& =1-\exp _{\frac{1}{2-q}}\left[-\lambda(2-q) t^{\beta}\right] .
\end{aligned}
$$


Let $\ln _{q}(x)$ denote the inverse function of $\exp _{q}(x)$, that is

$$
\ln _{q}(x)=\frac{x^{1-q}-1}{1-q}
$$

By (7), we have

$$
\ln _{\frac{1}{2-q}}\left(1-F_{q}\right)=-\lambda(2-q) t^{\beta}
$$

By taking logarithm again,

$$
\ln \left[-\ln _{\frac{1}{2-q}}\left(1-F_{q}\right)\right]=\ln \lambda+\ln (2-q)+\beta \ln t
$$

Let $y=\ln \left[-\ln _{\frac{1}{2-q}}\left(1-F_{q}\right)\right]$ and $x=\ln t$, then we have the linear form

$$
y=\beta x+\ln \lambda+\ln (2-q) .
$$

Let $t_{(1)} \leq \cdots \leq t_{(n)}$ be the order statistics corresponding to $t_{1}, \ldots, t_{n}$. Estimate the CDF $F_{q}$ by

$$
\widehat{F}_{q}\left(t_{(i)}\right)=\frac{i-0.3}{n+0.4}
$$

according to the Bernard's median-rank estimator [19] based on the rank of order statistics. Then the LSEs $\widehat{q}_{l}, \widehat{\beta}_{l}$ and $\widehat{\lambda}_{l}$ can be obtained by the following constrained optimization problem

$$
\begin{array}{cc}
\min & \sum_{i=1}^{n}\left[\widehat{y}_{i}-\beta x_{i}-\ln \lambda-\ln (2-q)\right]^{2} \\
\text { s.t. } & q<2, \\
\lambda>0, \\
\beta>0,
\end{array}
$$

where

$$
\widehat{y}_{i}=\ln \left\{-\ln _{\frac{1}{2-q}}\left[1-\widehat{F}_{q}\left(t_{(i)}\right)\right]\right\}, x_{i}=\ln t_{(i)} .
$$

As in Section 2.2, it is difficult to prove the uniqueness of the solution to the optimization problem (11). An optimization algorithm described later on was applied to solve this optimization problem. Execution of the algorithm for a wide range of initial values of the parameters showed that the algorithm always converged and the solution was unique. The initial values considered corresponded to all combinations of $q=0,0.2, \ldots, 1.8, \lambda=1,2, \ldots, 10$ and $\beta=1,2, \ldots, 10$.

\section{The CIs for the q-Weibull parameters}

\subsection{The asymptotic CIs}

The Fisher information matrix is frequently used to construct CIs for parameters based on asymptotic normality of the MLEs. Assume the parameter $\Theta$ is of interest. Under certain regularity conditions, the MLEs $\widehat{\Theta}$ follow the normal distribution $N\left(\Theta, I^{-1}(\Theta)\right)$ as $n \rightarrow \infty$, where $I^{-1}(\Theta)$ denotes the inverse matrix of the Fisher information matrix. The elements in the Fisher information matrix are the negatives of the expected values of the second order derivatives of $\ln L$ in (5), that is

$$
I(q, \beta, \lambda)=-E\left[\begin{array}{ccc}
\frac{\partial^{2} \ln L}{\partial q^{2}} & \frac{\partial^{2} \ln L}{\partial q \partial \beta} & \frac{\partial^{2} \ln L}{\partial q \partial \lambda} \\
\frac{\partial^{2} \ln L}{\partial \beta \partial q} & \frac{\partial^{2} \ln L}{\partial \beta^{2}} & \frac{\partial^{2} \ln L}{\partial \beta \partial \lambda} \\
\frac{\partial^{2} \ln L}{\partial \lambda \partial q} & \frac{\partial^{2} \ln L}{\partial \lambda \partial \beta} & \frac{\partial^{2} \ln L}{\partial \lambda^{2}}
\end{array}\right] .
$$


If the expected values are difficult to derive, they can be approximated by the negatives of the second order derivatives of $\ln L$ evaluated at the MLEs. $[9,20]$. Xu et al. [10] obtained the CIs for the q-Weibull parameters according to this approximation. Here, we derive the expected values.

The second order derivatives of $\ln L$ are

$$
\begin{aligned}
\frac{\partial^{2} \ln L}{\partial q^{2}}= & -\frac{n}{(q-2)^{2}}+\frac{2 \lambda}{(1-q)^{2}} \sum_{i=1}^{n} \frac{t_{i}^{\beta}}{1-(1-q) \lambda t_{i}^{\beta}}-\frac{\lambda^{2}}{1-q} \sum_{i=1}^{n} \frac{t_{i}^{2 \beta}}{\left[1-(1-q) \lambda t_{i}^{\beta}\right]^{2}} \\
& +\frac{2}{(1-q)^{3}} \sum_{i=1}^{n} \ln \left[1-(1-q) \lambda t_{i}^{\beta}\right] \\
\frac{\partial^{2} \ln L}{\partial q \partial \beta}= & \sum_{i=1}^{n} \frac{\lambda^{2} t_{i}^{2 \beta} \ln t_{i}}{\left[1-(1-q) \lambda t_{i}^{\beta}\right]^{2}} \\
\frac{\partial^{2} \ln L}{\partial q \partial \lambda}= & \sum_{i=1}^{n} \frac{\lambda t_{i}^{2 \beta}}{\left[1-(1-q) \lambda t_{i}^{\beta}\right]^{2}} \\
\frac{\partial^{2} \ln L}{\partial \beta^{2}}= & -\frac{n}{\beta^{2}}-\sum_{i=1}^{n} \frac{\lambda t_{i}^{\beta} \ln ^{2} t_{i}}{\left[1-(1-q) \lambda t_{i}^{\beta}\right]^{2}} \\
\frac{\partial^{2} \ln L}{\partial \beta \partial \lambda}= & -\sum_{i=1}^{n} \frac{t_{i}^{\beta} \ln t_{i}}{\left[1-(1-q) \lambda t_{i}^{\beta}\right]^{2}} \\
\frac{\partial^{2} \ln L}{\partial \lambda^{2}}= & -\frac{n}{\lambda^{2}}-\sum_{i=1}^{n} \frac{(1-q) t_{i}^{2 \beta}}{\left[1-(1-q) \lambda t_{i}^{\beta}\right]^{2}} .
\end{aligned}
$$

If $0<q<1$,

$$
\begin{aligned}
E\left(\frac{t^{2 \beta}}{\left[1-(1-q) \lambda t^{\beta}\right]^{2}}\right) & =\frac{2-q}{(1-q)^{3} \lambda^{2}} B\left(3, \frac{q}{1-q}\right) \\
E\left(\frac{t^{\beta}}{1-(1-q) \lambda t^{\beta}}\right) & =\frac{2-q}{(1-q)^{2} \lambda} B\left(2, \frac{1}{1-q}\right), \\
E\left(\frac{t^{\beta} \ln t}{\left[1-(1-q) \lambda t^{\beta}\right]^{2}}\right) & =\frac{2-q}{(1-q)^{2} \lambda \beta} B\left(2, \frac{q}{1-q}\right)(C 1-C 2), \\
E\left(\frac{t^{2 \beta} \ln t}{\left[1-(1-q) \lambda t^{\beta}\right]^{2}}\right) & =\frac{2-q}{(1-q)^{3} \lambda^{2} \beta} B\left(3, \frac{q}{1-q}\right)(C 3-C 2), \\
E\left(\frac{t^{\beta} \ln t}{\left[1-(1-q) \lambda t^{\beta}\right]^{2}}\right) & =\frac{2-q}{(1-q)^{2} \lambda \beta^{2}} B\left(2, \frac{q}{1-q}\right)\left[(C 1-C 2)^{2}+C 4\right], \\
E\left(\ln \left[1-(1-q) \lambda t^{\beta}\right]\right) & =\frac{q-1}{2-q},
\end{aligned}
$$

where

$$
\begin{aligned}
C 1 & =\psi^{(1)}(2)-\psi^{(1)}\left(2+\frac{q}{1-q}\right), \\
C 2 & =\ln \lambda+\ln (1-q), \\
C 3 & =\psi^{(1)}(3)-\psi^{(1)}\left(3+\frac{q}{1-q}\right), \\
C 4 & =\psi^{(2)}(2)-\psi^{(2)}\left(2+\frac{q}{1-q}\right), \\
\psi^{(k)}(x) & =\frac{d^{k} \ln \Gamma(x)}{d x^{k}} .
\end{aligned}
$$


Hence, the Fisher information matrix is

$$
I(q, \beta, \lambda)=n\left[\begin{array}{lll}
E_{11} & E_{12} & E_{13} \\
E_{21} & E_{22} & E_{23} \\
E_{31} & E_{32} & E_{33}
\end{array}\right]
$$

where

$$
\begin{aligned}
& E_{11}=\frac{1}{(q-2)^{2}}+\frac{2}{(1-q)^{2}(2-q)}-\frac{2-q}{(1-q)^{4}}\left[2 B\left(2, \frac{1}{1-q}\right)-B\left(3, \frac{q}{1-q}\right)\right] \\
& E_{12}=E_{21}=\frac{2-q}{(1-q)^{3} \beta} B\left(3, \frac{q}{1-q}\right)(C 2-C 3) \\
& E_{13}=E_{31}=-\frac{2-q}{(1-q)^{3} \lambda} B\left(3, \frac{q}{1-q}\right) \\
& E_{22}=\frac{1}{\beta^{2}}+\frac{2-q}{(1-q)^{3} \beta^{2}} B\left(2, \frac{q}{1-q}\right)\left[(C 1-C 2)^{2}+C 4\right] \\
& E_{23}=E_{32}=\frac{2-q}{(1-q)^{2} \lambda \beta} B\left(2, \frac{q}{1-q}\right)(C 1-C 2) \\
& E_{33}=\frac{1}{\lambda^{2}}+\frac{2-q}{(1-q)^{2} \lambda^{2}} B\left(3, \frac{q}{1-q}\right) .
\end{aligned}
$$

If $1<q<2$,

$$
\begin{aligned}
E\left(\frac{t^{2 \beta}}{\left[1-(1-q) \lambda t^{\beta}\right]^{2}}\right) & =\frac{2-q}{(q-1)^{3} \lambda^{2}} B\left(3, \frac{2-q}{q-1}\right), \\
E\left(\frac{t^{\beta}}{1-(1-q) \lambda t^{\beta}}\right) & =\frac{2-q}{(q-1)^{2} \lambda} B\left(2, \frac{2-q}{q-1}\right), \\
E\left(\frac{t^{\beta} \ln t}{\left[1-(1-q) \lambda t^{\beta}\right]^{2}}\right) & =\frac{2-q}{(q-1)^{2} \lambda \beta} B\left(2, \frac{1}{q-1}\right)(C 5-C 6), \\
E\left(\frac{t^{2 \beta} \ln t}{\left[1-(1-q) \lambda t^{\beta}\right]^{2}}\right) & =\frac{2-q}{(q-1)^{3} \lambda^{2} \beta} B\left(3, \frac{2-q}{q-1}\right)(C 7-C 6), \\
E\left(\frac{t^{\beta} \ln { }^{2} t}{\left[1-(1-q) \lambda t^{\beta}\right]^{2}}\right) & =\frac{2-q}{(q-1)^{2} \lambda \beta^{2}} B\left(2, \frac{1}{q-1}\right)\left[(C 5-C 6)^{2}+C 8\right], \\
E\left(\ln \left[1-(1-q) \lambda t^{\beta}\right]\right) & =\frac{2-q}{q-1},
\end{aligned}
$$

where

$$
\begin{aligned}
C 5 & =\psi^{(1)}(2)-\psi^{(1)}\left(\frac{1}{q-1}\right), \\
C 6 & =\ln \lambda+\ln (q-1), \\
C 7 & =\psi^{(1)}(3)-\psi^{(1)}\left(\frac{2-q}{q-1}\right), \\
C 8 & =\psi^{(2)}(2)+\psi^{(2)}\left(\frac{1}{q-1}\right) .
\end{aligned}
$$

Therefore, the Fisher information matrix is

$$
I(q, \beta, \lambda)=n\left[\begin{array}{lll}
E_{11} & E_{12} & E_{13} \\
E_{21} & E_{22} & E_{23} \\
E_{31} & E_{32} & E_{33}
\end{array}\right],
$$


where

$$
\begin{aligned}
& E_{11}=\frac{1}{(q-2)^{2}}-\frac{2(2-q)}{(1-q)^{4}}\left[B\left(2, \frac{2-q}{q-1}\right)-\frac{1}{q}-1+q\right], \\
& E_{12}=E_{21}=\frac{2-q}{(q-1)^{3} \beta} B\left(3, \frac{2-q}{q-1}\right)(C 7-C 6), \\
& E_{13}=E_{31}=-\frac{2-q}{(q-1)^{3} \lambda} B\left(3, \frac{2-q}{q-1}\right), \\
& E_{22}=\frac{1}{\beta^{2}}+\frac{2-q}{(q-1)^{2} \beta^{2}} B\left(2, \frac{1}{q-1}\right)\left[(C 5-C 6)^{2}+C 8\right], \\
& E_{23}=E_{32}=\frac{2-q}{(q-1)^{2} \lambda \beta} B\left(2, \frac{1}{q-1}\right)(C 5-C 6), \\
& E_{33}=\frac{1}{\lambda^{2}}-\frac{2-q}{(q-1)^{2} \lambda^{2}} B\left(3, \frac{2-q}{q-1}\right) .
\end{aligned}
$$

If $q=1$, the Fisher information matrix is

$$
\begin{aligned}
& I(\beta, \lambda)=-E\left[\begin{array}{ll}
\frac{\partial^{2} \ln L}{\partial \beta^{2}} & \frac{\partial^{2} \ln L}{\partial \beta \partial \lambda} \\
\frac{\partial^{2} \ln L}{\partial \beta \partial \lambda} & \frac{\partial^{2} \ln L}{\partial \lambda^{2}}
\end{array}\right] \\
& =\frac{n}{\beta^{2} \lambda^{2}}\left[\begin{array}{cc}
\lambda^{2}\left[\psi^{(2)}(1)+\left(1+\psi^{(1)}(1)-\ln \lambda\right)^{2}\right. \\
\lambda \beta\left(1+\psi^{(1)}(1)-\ln \lambda\right) & \lambda \beta\left(1+\psi^{(1)}(1)-\ln \lambda\right) \\
\beta^{2}
\end{array}\right] \text {. }
\end{aligned}
$$

The remaining case is $q \leq 0$. The arguments of the beta function $B(x, y)$ must satisfy $x>0$ and $y>0$. So, in this case, the beta function is no longer applicable and the expectations are intractable. Hence, we approximate the expected values by the negatives of the second order derivatives evaluated at the MLEs $\widehat{q}, \widehat{\beta}$ and $\widehat{\lambda}$ : that is,

$$
I_{\text {app }}(\widehat{q}, \widehat{\beta}, \widehat{\lambda})=-\left[\begin{array}{ccc}
\frac{\partial^{2} \ln L}{\partial q^{2}} & \frac{\partial^{2} \ln L}{\partial q \partial \beta} & \frac{\partial^{2} \ln L}{\partial q \partial \lambda} \\
\frac{\partial^{2} \ln L}{\partial \beta \partial q} & \frac{\partial^{2} \ln L}{\partial \beta^{2}} & \frac{\partial^{2} \ln L}{\partial \beta \partial \lambda} \\
\frac{\partial^{2} \ln L}{\partial \lambda \partial q} & \frac{\partial^{2} \ln L}{\partial \lambda \partial \beta} & \frac{\partial^{2} \ln L}{\partial \lambda^{2}}
\end{array}\right]
$$

evaluated at $q=\widehat{q}, \beta=\widehat{\beta}$ and $\lambda=\widehat{\lambda}$.

The covariance matrix of the MLEs is the inverse matrix of the Fisher information matrix, that is

$$
C=I^{-1}(q, \beta, \lambda)=\left[\begin{array}{lll}
c_{11} & c_{12} & c_{13} \\
c_{21} & c_{22} & c_{23} \\
c_{31} & c_{32} & c_{33}
\end{array}\right] .
$$

Hence, the $100(1-\alpha)$ percent asymptotic CIs for $q, \beta$ and $\lambda$ are

$$
\begin{aligned}
& {\left[\widehat{q}_{m}+U_{\frac{\alpha}{2}} \sqrt{c_{11}}, \min \left(2, \widehat{q}_{m}-U_{\frac{\alpha}{2}} \sqrt{c_{11}}\right)\right],} \\
& {\left[\max \left(0, \widehat{\beta}_{m}+U_{\frac{\alpha}{2}} \sqrt{c_{22}}\right), \widehat{\beta}_{m}-U_{\frac{\alpha}{2}} \sqrt{c_{22}}\right],} \\
& {\left[\max \left(0, \widehat{\lambda}_{m}+U_{\frac{\alpha}{2}} \sqrt{c_{33}}\right), \widehat{\lambda}_{m}-U_{\frac{\alpha}{2}} \sqrt{c_{33}}\right],}
\end{aligned}
$$

respectively, where $\widehat{q}_{m}, \widehat{\beta}_{m}, \widehat{\lambda}_{m}$ are the MLEs and $U_{\alpha}$ is the $\alpha$-quantile of the standard normal distribution. Here, the max and min operations are used to ensure that $\beta>0, \lambda>0$ and $q<2$. 


\subsection{The bootstrap CIs}

The bootstrap method is commonly used in applications. Suppose that the parameter of interest is $\theta$ and let $\widehat{\theta}$ denotes its estimate. The idea of the bootstrap method is to infer on $\theta$ using the distribution of $\widehat{\theta}$ according to a bootstrap sample. The bootstrap method could be classified into parametric and non-parametric bootstrap methods [10]. In this paper, a parametric bootstrap method is adopted to construct percentile bootstrap CIs [?, ?]. To construct the bootstrap CI for the q-Weibull parameters, the following algorithm is proposed to generate the bootstrap sample. Note that different bootstrap CIs could be obtained based on the MLEs and LSEs.

\section{Algorithm}

Given the MLEs (respectively, LSEs) $\widehat{q}_{m}, \widehat{\beta}_{m}, \widehat{\lambda}_{m}$ of the q-Weibull parameters and bootstrap sample size $B$ :

1: Generate a random sample $t_{1}^{b}, \ldots, t_{n}^{b}$ using the MLEs (respectively, LSEs) $\widehat{q}_{m}, \widehat{\beta}_{m}, \widehat{\lambda}_{m}$ through

$$
t^{b}=\left[\frac{1-(1-u)^{\frac{1-q}{2-q}}}{\lambda(1-q)}\right]^{\frac{1}{\beta}}
$$

where $u \sim$ Uniform $(0,1)$.

2: Based on the simulated sample $t_{1}^{b}, \ldots, t_{n}^{b}$, use (6) (respectively, (11)) to compute the bootstrap estimates of the q-Weibull parameters, say $\widehat{q}^{b}, \widehat{\beta}^{b}, \widehat{\lambda}^{b}$.

3: Repeat steps 1 and $2 B$ times and sort the obtained bootstrap estimates as $\widehat{q}_{1}^{b}<\cdots<\widehat{q}_{B}^{b}, \widehat{\beta}_{1}^{b}<\cdots<\widehat{\beta}_{B}^{b}$ and $\widehat{\lambda}_{1}^{b}<\cdots<\widehat{\lambda}_{B}^{b}$. The $100(1-\alpha) \%$ bootstrap CIs based on the MLEs (respectively, LSEs) for $q, \beta$ and $\lambda$ are

$$
\left[\widehat{q}_{\frac{B \alpha}{2}}^{b}, \widehat{q}_{B-\frac{B \alpha}{2}}^{b}\right],\left[\begin{array}{ll}
\widehat{\beta}_{\frac{B \alpha}{2}}^{b}, & \widehat{\beta}_{B-\frac{B \alpha}{2}}^{b}
\end{array}\right],\left[\begin{array}{ll}
\widehat{\lambda}_{\frac{B \alpha}{2}}^{b}, & \widehat{\lambda}_{B-\frac{B \alpha}{2}}^{b}
\end{array}\right],
$$

respectively.

\section{The simulation study}

A Monte Carlo simulation under different parameter settings is carried out in this section to compare the performance of the MLEs and LSEs as well as the asymptotic and bootstrap CIs for the q-Weibull parameters. To conduct this study, settings for the q-Weibull parameters $q, \beta, \lambda$ and the sample size $n$ are required. Without loss of generality, we set $\lambda=1$. We take $n$ to be $100,200,500,800$ and 1000 . For the values of $\beta$ and $\lambda$, we refer to [10]. The settings are tabulated in Table 1 . Besides, to cover all the ranges of $q$ and $\beta$, the cases of $q=-1, \beta=0.5$ and $q=-1, \beta=1.5$ are also considered.

Table 1. The settings for the parameters $q$ and $\beta$.

\begin{tabular}{ccc}
\hline$q$ & $\beta$ & Behavior of hazard rate function \\
\hline 0.5 & 0.5 & Bathtub-shaped \\
1.5 & 0.5 & Decreasing \\
0.5 & 1.5 & Increasing \\
1.5 & 1.5 & Unimodal \\
\hline
\end{tabular}

Under each parameter setting, a sample of size $n$ was generated using (18). Using this random sample, the MLEs $\widehat{q}_{m}, \widehat{\beta}_{m}$, $\widehat{\lambda}_{m}$ and the LSEs $\widehat{q}_{l}, \widehat{\beta}_{l}, \widehat{\lambda}_{l}$ were calculated based on (6) and (11). For the problem (6), the optimization function fmincon in the Matlab software with 'interior-point' algorithm was used to obtain the MLEs. For the problem (11), the optimization function Isqnonlin in the Matlab software with 'trust-region-reflective' algorithm was used to compute the LSEs. Next, the asymptotic 90\% CIs were derived through (17) by treating the MLEs of the q-Weibull parameters as the true values. In addition, 90\% bootstrap CIs based on the MLEs and LSEs were obtained by running the algorithm with $B=5000$. We replicated this process 10000 times for each parameter setting. To compare the MLEs and LSEs, the biases and mean squared errors (MSEs) were estimated. Similarly, the coverage probabilities (CPs) and average interval widths of the three CIs were computed for comparison. For ease of illustration, the relative bias, relative MSE and relative average width were obtained as the ratios of bias, MSE and average width to the corresponding true values. They are depicted in Figs. 1 - 2. 

(a) $(q, \beta)=(0.5,0.5)$
(b) $(q, \beta)=(0.5,1.5)$
(c) $(q, \beta)=(1.5,0.5)$
(d) $(q, \beta)=(1.5,1.5)$
(e) $(q, \beta)=(-1,0.5)$
(f) $(q, \beta)=(-1,1.5)$

Figure 1. The comparisons between the MLEs and LSEs: MLEs (solid line) and LSEs (line with 'o').
(a) $(q, \beta)=(0.5,0.5)$
(b) $(q, \beta)=(0.5,1.5)$
(c) $(q, \beta)=(1.5,0.5)$
(d) $(q, \beta)=(1.5,1.5)$
(e) $(q, \beta)=(-1,0.5)$
(f) $(q, \beta)=(-1,1.5)$

Figure 2. The comparisons among the three CIs: bootstrap CI based on the MLEs (solid line), bootstrap CI based on the LSEs (line with '+') and asymptotic CI (line with 'o').

From Figs. 1 - 2, we can observe the following.

For comparison of the MLEs and the LSEs:

1. When $q=0.5$,

(1) The performance of $\widehat{q}_{l}$ is poor as the bias and MSE are both large.

(2) For $\beta$ and $\lambda$, the absolute biases and MSEs of $\widehat{\beta}_{l}, \widehat{\lambda}_{l}$ are greater than those of $\widehat{\beta}_{m}, \widehat{\lambda}_{m}$.

2. When $q=1.5$,

(1) Both the MLEs and the LSEs perform well.

(2) The MSEs of the MLEs and the LSEs are close.

(3) In terms of absolute bias, $\widehat{q}_{m}$ is less than $\widehat{q}_{l}, \widehat{\beta}_{m}$ is similar to $\widehat{\beta}_{l}$ and $\widehat{\lambda}_{m}$ is larger than $\widehat{\lambda}_{l}$ for $\beta=0.5$. In terms of absolute bias, $\widehat{q}_{m}$ is less than $\widehat{q}_{l}, \widehat{\beta}_{m}$ is similar to $\widehat{\beta}_{l}$ and $\widehat{\lambda}_{m}$ is close to $\widehat{\lambda}_{l}$ for $\beta=1.5$.

3. When $q=-1$,

(1) The performance of $\widehat{q}_{l}$ is poor as the bias and MSE are both unacceptable especially the MSE.

(2) The absolute bias and MSE of $\widehat{\beta}_{m}$ are both smaller than those of $\widehat{\beta}_{l}$.

(3) The absolute bias of $\widehat{\lambda}_{m}$ is greater than that of $\widehat{\lambda}_{l}$ for $\beta=0.5$. The absolute bias of $\widehat{\lambda}_{m}$ is close to that of $\widehat{\lambda}_{l}$ for $\beta=1.5$. But the MSE of $\widehat{\lambda}_{m}$ is always less than that of $\widehat{\lambda}_{l}$.

For comparison of the three CIs:

1. The CP of the asymptotic CI is always the smallest. But it is far from the nominal level except when $q=0.5$ with small sample size. The reason may be that the average interval width of asymptotic CIs is narrowest. In the case of $q=0.5$ with small sample size, the average interval widths become large making the CP better. The sudden increase is possibly caused by the large bias of the MLE $\widehat{q}_{m}$.

2. When $q=0.5$,

(1) The CPs of the two bootstrap CIs agree with the nominal level. The CP of the bootstrap CI based on the MLEs is better than that based on the LSEs.

(2) The average interval width of the bootstrap CI based on the MLEs is narrower than that based on the LSEs.

(3) The average interval width of the bootstrap CI based on the LSEs for $q$ is worse, which may be due to the poor performance of the LSE $\widehat{q}_{l}$.

2. When $q=1.5$,

(1) The CPs of the two bootstrap CIs coincide well with the nominal level. Further, the CP of the bootstrap CI based on the MLEs is superior to that based on the LSEs.

(2) The differences between the average interval widths of the two bootstrap CIs (based on the MLEs and LSEs) are tiny.

3. When $q=-1$,

(1) For $q$, when the sample size $n$ is small, the CP of the bootstrap CI based on the LSEs is largest while the CP of the bootstrap CI based on the MLEs is smallest. But with $n$ increasing, the CPs of the two bootstrap CIs become close. For the average interval width, the bootstrap CI based on the MLEs is always narrower.

(2) For $\beta$, the CPs of the two bootstrap CIs are similar. However, the average interval width of the bootstrap CI based on the MLEs is narrower than that based on the LSEs.

(3) For $\lambda$, when the sample size is small, the CP of the bootstrap CI based on the MLEs is less than that based on the LSEs. They become close with $n$ increasing. But the average interval width of the bootstrap CI based on the MLEs is narrower. 
The estimate with the smallest bias and the smallest MSE should be selected as the point estimate. In terms of CP and average interval width, the $\mathrm{CI}$ having the $\mathrm{CP}$ around the nominal level and the narrowest width is superior. Based on the simulation results, we recommend the MLEs and bootstrap CIs based on the MLEs for the q-Weibull parameters. In addition, the results would be more robust if larger data sets were collected.

The regularity conditions for asymptotic normality should be checked for the use of asymptotic CIs. These conditions are often hard to check analytically. We checked multivariate normality of the simulated sample of estimates $(\widehat{q}, \widehat{\lambda}, \widehat{\beta})$ for each $n=100,200,500,800,1000$. The test for multivariate normality was performed using the R package MVN. The $p$ value of the test for each $n$ was above 0.05 . Hence, it is reasonable to assume that normality of $(\widehat{q}, \widehat{\lambda}, \widehat{\beta})$ holds for $n \geq 100$.

\section{Illustrative example}

In this section, an illustrative example is provided. A published dataset about the times to first failure for a group of 36 generators of 500MW [10] is used. The data are $0.058,0.070,0.090,0.105,0.113,0.121,0.153,0.159,0.224,0.421,0.570$, 0.596, 0.618, 0.834, 1.019, 1.104, 1.497, 2.027, 2.234, 2.372, 2.433, 2.505, 2.690, 2.877, 2.879, 3.166, 3.455, 3.551, 4.378, $4.872,5.085,5.272,5.341,8.952,9.188$ and 11.399 (1000's of hours).

First, a model should be selected to describe the data. We chose the Weibull and q-Weibull distributions for comparison. After estimating $\widehat{F}_{q}$ through (10), this data set was fitted by the q-Weibull distribution through (9) and the Weibull distribution by the Weibull probability plot method [19]. The probability plots are shown in Fig. 3. It is difficult to distinguish the two distributions from Fig. 3 as they both seem to fit the data well. But the sums of the errors resulted from the fitting are 2.98 and 3.11 for the q-Weibull and Weibull distributions, respectively. Besides, the log-likelihoods are -68.06 and -68.69 , respectively. Hence, the q-Weibull distribution should be preferred to the Weibull distribution.

Figure 3. The probability plots for the fits of the q-Weibull and Weibull distributions.

Next, the MLEs and LSEs of the q-Weibull parameters are computed based on (6) and (11). For the two optimization problems, the initial values are all set to be 1 (although the results were the same for a wide range of other initial values). The optimization methods for the two problems are similar to those in the simulation study. They were both solved by the Matlab software. The optimization function fmincon with 'interior-point' algorithm was used for the problem (6). The optimization function lsqnonlin with 'trust-region-reflective' algorithm was used for the problem (11).

Further, different CIs were constructed. For the asymptotic CI, the MLE $q_{m}=0.4318$. Through (12) and treating the MLEs as the true values, the Fisher information matrix could be obtained as

$$
I(q, \beta, \lambda)=\left[\begin{array}{ccc}
227.28 & -390.17 & -590.51 \\
-390.17 & 736.91 & 988.30 \\
-590.50 & 988.30 & 163.98
\end{array}\right] .
$$

Then the asymptotic CI is yielded by (17). Next, after running the algorithm with $B=5000$, the two bootstrap CIs based on the MLEs and LSEs were constructed using (19). For ease of comparison, the interval widths of the three CIs were also computed. All the results are tabulated in Table 2.

Table 2. The parameter estimates and CIs.

\begin{tabular}{cccccc}
\hline \multirow{2}{*}{ MLEs } & \multirow{2}{*}{ LSEs } & \multirow{2}{*}{ asymptotic CI } & \multicolumn{2}{c}{ bootstrap CI } \\
\cline { 5 - 6 } & & & MLEs & LSEs \\
\hline$q$ & 0.4318 & 0.6508 & {$[0.1716,0.6921](0.5204)$} & {$[-76.8152,1.0316](77.8468)$} & {$[-103.2563,1.1776](104.434)$} \\
$\beta$ & 0.6697 & 0.6884 & {$[0.6427,0.6956](0.0539)$} & {$[0.4363,0.9858](0.5494)$} & {$[0.4031,0.9963](0.5932)$} \\
$\lambda$ & 0.2824 & 0.3484 & {$[0.2652,0.2995](0.0343)$} & {$[0.0046,0.5556](0.5509)$} & {$[0.0034,0.7336](0.7302)$} \\
\hline
\end{tabular}

The MLEs are the same as the ones in [10]. The MLEs and LSEs of $\beta$ and $\lambda$ are similar. But the MLE and LSE of $q$ are different. Among the three CIs, the asymptotic CI is the narrowest. The width of the bootstrap CI based on the MLEs is smaller than that based on the LSEs.

These results agree well with the simulation results. In the simulation study, the narrowest of the CIs, the asymptotic CI, had a CP is different from the nominal level. The asymptotic CI here covers the corresponding MLE, but whether it covers 
the true value of the q-Weibull parameters is hard to know. Since the CP of the bootstrap CI based on the MLEs coincides with the nominal level well, the bootstrap CI based on the MLEs should be selected as the CI for the q-Weibull parameters. In addition, the MLEs could be used as the point estimates of the q-Weibull parameters.

\section{Conclusions}

We have considered point as well as interval estimation of the q-Weibull parameters. The point estimates, the MLEs and LSEs, for the q-Weibull parameters are known in the literature. For interval estimation, we have proposed three new estimates: asymptotic CIs based on the MLEs, bootstrap CIs based on the MLEs and bootstrap CIs based on the LSEs. For the asymptotic CIs, explicit expressions have been derived for the Fisher information matrix.

We have compared the performances of the different point estimates (and the different interval estimates) by a simulation study and a real data application. The point estimates were compared in terms of bias and mean squared error. The interval estimates were compared in terms of coverage probabilities and average interval widths. The MLEs are shown to perform better than the LSEs in terms of bias and mean squared error. The bootstrap CIs based on the MLEs are shown to perform better than others in terms of coverage probabilities and average interval widths.

\section{Acknowledgment}

This work was partially supported by the National Natural Science Foundation of China under grant nos.71571188 and 61573370. The authors would like to thank the two referees and the Editor for careful reading and comments which greatly improved the paper.

\section{References}

[1] Sumiyoshi Abe and Yuko Okamoto. Nonextensive statistical mechanics and its applications, volume 560. Springer, 2001.

[2] Murray Gell-Mann and Constantino Tsallis. Nonextensive entropy: Interdisciplinary applications. Oxford University Press, 2004.

[3] S Picoli Jr, RS Mendes, LC Malacarne, and RPB Santos. q-distributions in complex systems: A brief review. Brazilian Journal of Physics, 39(2A):468-474, 2009.

[4] S Picoli Jr, RS Mendes, and LC Malacarne. q-exponential, weibull, and q-weibull distributions: An empirical analysis. Physica A: Statistical Mechanics and Its Applications, 324(3):678-688, 2003.

[5] Saralees Nadarajah and Samuel Kotz. On the q-type distributions. Physica A: Statistical Mechanics and Its Applications, 377(2):465-468, 2007.

[6] Evandro Marcos Saidel Ribeiro and Gilberto Aparecido Prataviera. Modeling empirical distributions of firm size with q-distributions. International Journal of Applied Mathematics, 28(6):715-725, 2015.

[7] Edilson M Assis, Ernesto P Borges, and Silvio AB Vieira de Melo. Generalized q-weibull model and the bathtub curve. International Journal of Quality and Reliability Management, 30(7):720-736, 2013.

[8] Xiang Jia, Ping Jiang, and Bo Guo. Reliability evaluation for weibull distribution under multiply type- censoring. Journal of Central South University, 22:3506-3511, 2015.

[9] Xiang Jia, Dong Wang, Ping Jiang, and Bo Guo. Inference on the reliability of weibull distribution with multiply type-i censored data. Reliability Engineering and System Safety, 150:171-181, 2016.

[10] Meng Xu, Enrique López Droguett, Isis Didier Lins, and Márcio das Chagas Moura. On the q-weibull distribution for reliability applications: An adaptive hybrid artificial bee colony algorithm for parameter estimation. Reliability Engineering and System Safety, 158:93-105, 2017.

[11] Saad J Almalki and Saralees Nadarajah. Modifications of the weibull distribution: A review. Reliability Engineering and System Safety, 124:32-55, 2014. 
[12] Tommi Vuorenmaa. A q-weibull autoregressive conditional duration model and threshold dependence. Technical report, Discussion Paper 117, University of Helsinky, 2006.

[13] Tommi A Vuorenmaa. A q-weibull autoregressive conditional duration model with an application to nyse and hse data. Available at SSRN 1952550, 2009.

[14] KK Jose and Shanoja R Naik. On the q-weibull distribution and its applications. Communications in Statistics-Theory and Methods, 38(6):912-926, 2009.

[15] KK Jose, Shanoja R Naik, and Miroslav M Ristić. Marshall-olkin q-weibull distribution and max-min processes. Statistical Papers, 51(4):837-851, 2010.

[16] UMS Costa, VN Freire, LC Malacarne, RS Mendes, S Picoli Jr, EA De Vasconcelos, and EF da Silva Jr. An improved description of the dielectric breakdown in oxides based on a generalized weibull distribution. Physica A: Statistical Mechanics and Its Applications, 361(1):209-215, 2006.

[17] Isabel Sartori, Edilson M de Assis, Adilton L da Silva, Rosana LF Vieira de Melo, Ernesto P Borges, et al. Reliability modeling of a natural gas recovery plant using q-weibull distribution. Computer Aided Chemical Engineering, 27:1797$1802,2009$.

[18] Edilson M Assis, Ernesto P Borges, Silvio AB Vieira de Melo, and Leizer Schnitman. Modeling failure rate of a robotic welding station using generalized q-distributions. International Journal of Quality and Reliability Management, 32(2):156-166, 2015.

[19] LF Zhang, Min Xie, and Loon Ching Tang. A study of two estimation approaches for parameters of weibull distribution based on wpp. Reliability Engineering and System Safety, 92(3):360-368, 2007.

[20] A Clifford Cohen. Maximum likelihood estimation in the weibull distribution based on complete and on censored samples. Technometrics, 7(4):579-588, 1965. 